This is the Pre-Published Version

\title{
A Unified Picture Of The Scaling And Non-Scaling Behavior In Quantum Hall Plateau Transitions
}

\author{
X. R. Wang ${ }^{1}$, G. Xiong ${ }^{2}$, and Q. Niu ${ }^{3}$ \\ ${ }^{1}$ Physics Department, The Hong Kong University of Science and Technology, Clear Water Bay, Hong Kong, China; \\ ${ }^{2}$ Physics Department, Beijing Normal University, Beijing 100875, China; ${ }^{3}$ Physics Department, The University of \\ Texas at Austin, Austin, Texas 78712-1081, USA
}

\begin{abstract}
A unified picture of the scaling and non-scaling behavior in quantum Hall plateau transitions is proposed. We show that the interband mixing of states with opposite chiralities and the degrees of the inter-Landau-band overlap are the key ingredients to the problem. We find that the mixing of states with opposite chiralities tends to delocalize electronic states, leading to the formation of extended-state band near the center of each Landau subband. However, this requires sufficient interband overlap to interlock the two sets of states. We find extended states in each Landau band evolve from its center to a narrow band around the center as the interband overlap increases. Thus the nature of a plateau transition changes from a continuous phase transition at a low interband overlap to a cross of a narrow metallic phase at a high interband overlap.
\end{abstract}

\section{INTRODUCTION}

One overlooked issue of the integer quantum Hall $(\mathrm{QH})$ effect is the nature of a plateau-to-plateau (P-P) transition. Existing theories [1] assume it to be a continuous quantum phase transition, which means an algebraic divergence of longitudinal or Hall resistance slope, and an algebraic shrinkage of longitudinal resistance peak at the transition point in temperature $T$. Such scaling behaviors were indeed observed in experiments on clean samples [2]. However, experiments [3] on relatively dirty samples show that the longitudinal resistance slope remains finite and the resistance peak width remains nonzero when extrapolated to $T=0$, i.e., a non-scaling behavior at P$\mathrm{P}$ transitions in contradiction with the expectation of continuous quantum phase transitions.

In this report, we present a unified explanation to both scaling and non-scaling behavior in the P-P transitions within the network description of $\mathrm{QH}$ systems. We show that interband mixing and interband overlap are two essential ingredients.

\section{MODEL, METHODS AND RESULTS}

According to the semi-classical theory[4], an electronic state of a disordered system in a strong magnetic field can be approximately decomposed into a rapid cyclotron motion and a slow drifting motion of the guiding center. The trajectory of the drifting motion of a guiding center is along an equipotential contour. Each contour corresponds to one quantum state represented by squares in Fig. 1. The drifting direction denoted by arrows is unidirectional. In the case of a significant overlap between two neighboring Landau bands (LB), interband mixing is possible, and two sets of states with opposite chiralities are needed if we consider an energy between two Landau levels. One set from the upper LB is located around valleys (denoted by letter $\mathrm{V}$ inside squares) while the other from the lower LB are around peaks (denoted by P).

Interband Mixing Induced Delocalization: In the absence of interband mixing, the model is reduced to two decoupled single-band models and all electronic states between the two LBs are localized [4]. If we introduce interband mixing, the localized contours (loops) may become less localized. To see this, let us consider an extreme case with no tunneling at saddle points, but with such a strong interband mixing that an electron will jump from a loop around a valley to its neighboring loop around a peak and vice versa, as shown by $\mathrm{B} \rightarrow \mathrm{C}$ in Fig. 1(a). Follow the trajectory of an electron starting at $\mathrm{A}$, it will be $\mathrm{A} \rightarrow \mathrm{B} \rightarrow \mathrm{C} \rightarrow$ $\mathrm{D} \rightarrow \mathrm{E} \ldots$... The electron is no longer confined on a closed loop, but delocalized! 


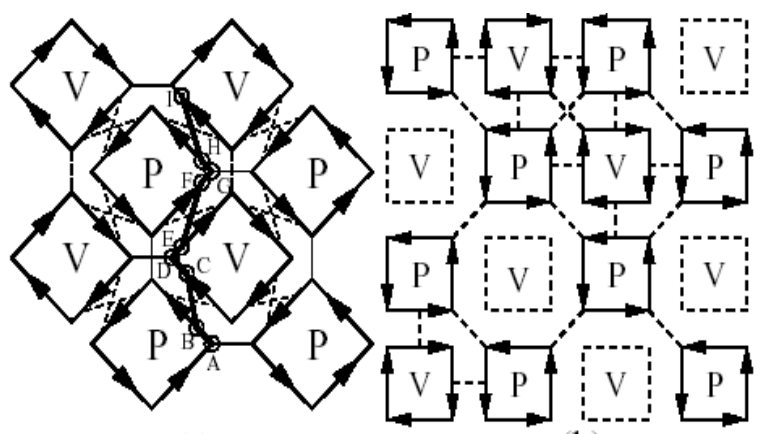

(a)

(b)

FIGURE 1 Schematic drawing of two channel network.

Diluted two channel network: To faithfully model the interband mixing effect on the states around the center of a particular LB, say the lower band, we need to notice that only a fraction $p$ of valleys provides upper-band loops because the rest of random potential valleys are too shallow while almost all loops around peaks should be present. The fraction of randomly selected occupied valleys $p$ can be used to measure the interband overlap around the lower band center. In Fig. $1 \mathrm{~b}$, the squares in the solid line are present, and those in dashed line are absent. According to our previous study [5], extended states around the lower band center form a narrow band due to a very weak interband mixing when $p=1$. We expect that each of these narrow bands shrink to a singular point as $p$ is tuned down to a critical value with a fixed value of weak interband mixing.

Methods of level statistics analysis: We use the approach in reference 6 to study the localization of the two-channel network, There are two wavefunction amplitudes, one for the upper and the other for the lower bands, on each link. Arrange them into a vector $\phi$. The dynamics of the network is described by an evolution operator $U(E)$ which depends on energy $E$, interband mixing strength $J$, and $p$. Its construction can be found in reference 5 . The eigenvalue equation of this operator is $U(E) \phi_{\alpha}(\mathrm{E})=\exp \left(i \omega_{\alpha}\right) \phi_{\alpha}(\mathrm{E})$, where $\alpha$ is the eigenstate index. The eigenenergies $E_{\mathrm{n}}$ of the system are those $E$ with $\omega_{\alpha}$ being an integer multiples of $2 \pi . I_{0}=\int s^{2} P(s) d s / 2$ is used to examine the localization property, where $P(s)$ is the level-spacing distribution function of the eigenenergies. If $I_{0}$ increases with system size $L$, the state is localized. Otherwise, it is extended.

Results: Fig. 2a-c are the curves of $I_{0}$ vs. $p$ for various energy $E$, interband mixing strength $J$, and system size $L$. $I_{0}-p$ curves of different $L$ with fixed $(E, J)$ cross through the same point $p_{\mathrm{c}}$. Thus each state exhibits a localization-delocalization transition at $p_{\mathrm{c}}$. The critical $p_{\mathrm{c}}$ increases with $E$. Fig. $2 \mathrm{~d}$ is the $p_{\mathrm{c}}$ vs. $E$. $p_{\mathrm{c}}$ approaches to a non-zero value as $E \rightarrow 0$ indicates that a certain fraction of states from the upper LB is needed in order to create an extended state between two Landau levels.

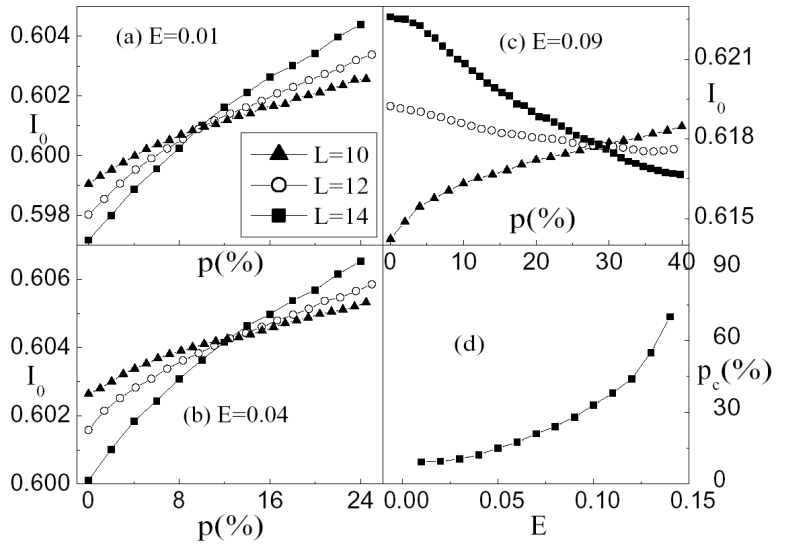

FIGURE 2 (a)-(c) are $I_{0}$ vs. $p$ for $L=10,12,14$ with $J=0.1$ and $E=0.01$ (a); $E=0.04$ (b), and $E=0.09$ (c). (d) is $p_{\mathrm{c}}$ vs. $E$ for $J=0.1$.

Summary: We find that there exists a critical value for the interband overlap. Above the value, extended states form bands around each LB center. A P-P transition is a cross through a narrow metallic phase, leading to a non-scaling behavior. Below the value, there is only a singular extended state in each LB. In this case, a P-P transition is a true continuous quantum phase transition with a scaling behavior.

\section{Acknowledgments}

This work was supported by RGC of HKSAR, China.

\section{REFERENCES}

1. S. Kivelson, D. H. Lee and S. C. Zhang, Phys. Rev. B 46, 2223(1992); D. Z. Liu, X. C. Xie and Q. Niu, Phys. Rev. Lett. 76, 975(1996); X. R. Wang, X. C. Xie, Q. Niu, and J. Jain, cond-mat/0008411.

2. H. P. Wei et. al., Phy. Rev. Lett. 61, 1294 (1988); H. W. Jiang et. al., Phys. Rev. Lett. 71, 1439(1993); I. Glozman et. al., Phys. Rev. Lett. 74, 594(1995); D. Shahar et. al., Phys. Rev. B 52, R14372(1995); S.-H. Song et. al., Phys. Rev. Lett. 78, 2200(1997); R. T. F. Schaijk et. al., Phys. Rev. Lett. $84,1567(2000)$.

3. M. Hilke et. al., Phy. Rev. B 56, 15545(1997); D. Shahar et. al., Solid State Commun. 102, 817(1997); M. Razeghi et. al., Solid State Commun. 107, 19(1998); A. A. Shashkin et. al., Phys. Rev. B 49, 14486(1994); N. Q. Babalan et. al., Phys. Rev. Lett. 81, 4967(1998).

4. J. T. Chalker and P. D. Coddington, J.Phys. C: Solid State Phys. 21, 2665(1988).

5. G. Xiong et. al., Phys. Rev. Lett. 87, 216802(2001).

6. R. Klesse and M. Metzler, Phys. Rev. Lett. 79, 721(1997); M. Metzler and I. Varga, J. Phys. Soc. Jpn. 67, 1856(1998). 